\title{
A screw based methodology for instantaneous dynamic balance
}

\author{
J.J. de Jong ${ }^{\mathrm{a}, *}$, J. van Dijk ${ }^{\mathrm{a}}$, J.L. Herder ${ }^{\mathrm{b}}$ \\ ${ }^{a}$ Laboratory of Precision Engineering, University of Twente, PO Box 217, 7500 AE Enschede, The Netherlands \\ ${ }^{b}$ Department of Precision and Microsystems Engineering, Delft University of Technology, Mekelweg 2, 2628 CD DELFT, The Netherlands
}

\begin{abstract}
Fast-moving industrial robots exert large varying reaction forces and moments on their base frame, inducing vibrations, wear and accuracy degeneration. These shaking forces and moments can be eliminated by a specific design of the mass distribution of the robot links, resulting in a dynamically balanced mechanism. Obtaining the conditions for dynamic balance proves to be a hurdle even for simple planar parallel mechanisms due to the required inclusion and inspection of the kinematic relations. In this paper, a screw theory based methodology is presented, which gives and solves the necessary instantaneous dynamic balance conditions for planar and spatial mechanisms in an uniform and geometrical manner. Instantaneous dynamic balance yields a pose in which robot accelerations induce no shaking forces and moments. This is interpreted as an intersection point of multiple reactionless paths. This method is applied to a 2-DOF planar mechanism, named the Fuga I, for which it resulted in two perpendicularly intersecting reactionless paths, intersecting in the middle of the workspace. Experiments on this demonstrator validated the instantaneous dynamic balance by showing a reduction of approximately $95 \%$ of the peak-to-peak shaking forces and moments over the intersecting reactionless paths.
\end{abstract}

Keywords: Dynamic balance, screw theory, parallel manipulator, spatial mechanisms, five-bar mechanism, reactionless path, experimental validation

\section{Introduction}

Industrial manipulators moving with high speeds and accelerations induce strong shaking forces and shaking moments at the base frame, causing disturbing vibrations in the frame and the surroundings [1]. These disrupting shaking forces and shaking moments can be eliminated by design of robot kinematics and inertial parameters, i.e. the mass, center of mass (COM), and moments of inertia (MOI) [2, 3], resulting in a dynamically balanced robot. When only the shaking forces are canceled the system is said to be force balanced, and moment balanced when the shaking moments are canceled [4]. Since the shaking forces and moments are the derivatives of the linear and angular momentum, dynamic balance is obtained when the linear and angular momentum are constant (or zero in practice) [5].

Commonly, force balance is considered prior to moment balance and is obtained by choice of counter-masses [6. 7]. Consecutively, moment balance is achieved by addition of reaction wheels [8], counter-mechanisms [9, 10] or idler loops [11], potentially leading to unfavorable complexity, additional mass and higher motor torques [4].

Fortunately, some parallel mechanisms, such the kite type and the anti-parallelogram type 4R planar four-bar, permit a dynamically balanced design without the need of additional counter-mechanisms [12]. Yet, the process to find these designs relies on manipulation and factorization of the dynamic balancing conditions, i.e the momentum equations, in minimal coordinates. The intrinsic complexity of the loop closure equations makes this manual process increasingly difficult for higher-DOF and spatial mechanisms. Therefore, Gosselin et al. partially automated the factorization process through toric geometry [13], and later algebraic geometry [14]. These algebraic methods still require some case-by-case treatment, and are yet to be extended to multi-DOF mechanisms. Furthermore, the

\footnotetext{
${ }^{*}$ Corresponding author

Email address: j.j.dejong@utwente.nl (J.J. de Jong)
} 
algebraic nature of the balance procedure hinders the derivation of intuition, which is desired from a designers point of view.

Synthesis methods partly overcome the inspection of equations through stacking and recombination of dynamically balanced elements. Ricard et al. [12], and later Wu et al. [15], used the dynamic balanced four-bar linkage as a building block for multi-DOF, planar and spatial, dynamic balanced mechanisms, resulting in rather complex structures with unfavorable mass to payload ratios [16]. Van der Wijk et al. developed Fishers principal vector method of describing human motion [17] into a synthesis method, which produces inherently force balance mechanisms [18]. Based on this method, the 3-DOF over-actuated DUAL V was presented [19]. Dynamic balance was achieved over two perpendicular paths by mirror symmetry of the design. Although this synthesis methodology yields new dynamically balanced mechanisms, it does not cover all the possible solutions [20], and is currently confined to planar linkages.

Alternatively, dynamically balanced behavior can be enforced by limiting the robot motion to reactionless paths [21, 22], or trajectories [23]. These methods are less restrictive on the choice of inertial parameters and enable 'dynamic balance when needed'. For example, during the traveling phase of a pick and place robot, when accuracy is not essential, the robot can follow any trajectory but just before and during the pick or placement phase the robot follows a reactionless path, in order to let vibrations die out and enable an accurate motion. Currently, only for specific kinematic structures it is known how to shape these reactionless paths by design of the robot [21, 19].

In pursuit of an intuitive method that yields all force and moment balanced design for arbitrary, planar or spatial linkages, we propose to use screw theory. In this paper, a screw theory based method is presented that yields instantaneous dynamic balance for arbitrary mechanisms. Instantaneous dynamic balance is a pose in which the accelerations of the robot will not induce shaking forces and moments. We will show that such an instantaneous pose is the intersection of multiple reactionless paths. This enables the design of reactionless paths by choice of this pose. The method relies on an unified, geometric interpretation of the angular and linear momentum, leading to a set of feasibility bounds on the selection of the dynamic balance solution. The validity of this method will be shown by design, construction and measurement of an experimental demonstrator named the Fuga I, which is completely force balanced over the whole workspace and moment balanced along two perpendicular paths, which intersect in the middle of the workspace.

The paper is organized as follows: Firstly, the screw theory with its application to rigid body dynamics will be recapitulated. The dynamic balance conditions are given in the screw theory framework. Secondly, the dynamic balance conditions are solved in two steps; A) on mechanism level - posing conditions on a specific number of the bodies, and B) on body level - solving these conditions to obtain a range of inertial parameters for these bodies. Thirdly, this method is applied to a five-bar demonstrator, and validated by both simulations and experiments. This paper is a generalization of the method presented in [24], which was confined to planar mechanisms consisting of revolute joints.

\section{Screw based dynamic balancing method}

Screw theory [25] provides a unified geometrical interpretation of the instantaneous spatial motion - termed twist - of a rigid body and of the force and torque - termed wrench - acting on this body. On one hand, screw theory leans on Mozzi-Chasles' theorem, which states that all rigid body motion can be interpreted as a rotation around an axis in space and a translation along that axis. Similarly, for forces and moments, Poinsot's theorem states that the sum of forces and moments acting on a body can be represented by a force along a line in space and a torque around that line. These spatial interpretations are termed screws. On the other hand, screw theory draws on Lie's theory of exponential mappings and manifolds - which can be interpreted as the configuration space of connected rigid bodies. This states that the admissible twists lie on the tangent space of this manifold. These screws are therefore differential and instantaneous properties. Screw theory has been applied to a wide variety of modeling and design problems including singularity analysis [26], kinematic synthesis methods [27], and robot dynamics [28, 29, 30]. The extension of screw theory to a dynamic balancing procedure for multi-DOF, closed-loop mechanisms has not been attempted.

\subsection{Screw theory}

The angular and linear velocity, respectively $\omega$ and $\mathbf{v}$ together form the twist $\mathbf{t}$ of a body. The twist is interpreted as the rotation around an instantaneous axis in the direction of $\omega$, passing through point $\mathbf{r}_{t}$ and a translation along this 


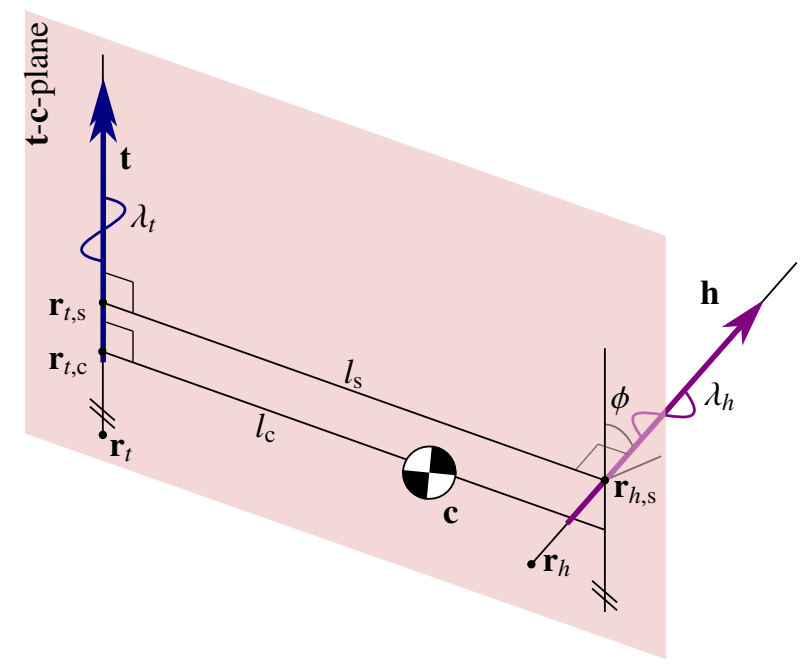

Figure 1: A geometrical representation of the momentum $\mathbf{h}$ generated by a twist $\mathbf{t}$ and body with a COM at $\mathbf{c}$.

axis, called the pitch $\lambda_{t}$ Fig. 1 . Therefore $\mathbf{v}$ is the velocity of the point passing through the origin

$$
\mathbf{t}=\left[\begin{array}{c}
\omega \\
\mathbf{v}
\end{array}\right] \quad \mathbf{v}=\mathbf{r}_{t} \times \omega+\lambda_{t} \omega
$$

From a given twist the axis location and the pitch can be computed as

$$
\mathbf{r}_{t}=\frac{\omega \times \mathbf{v}}{\|\omega\|^{2}}+\delta_{t} \omega \quad \lambda_{t}=\frac{\mathbf{v}^{\top} \omega}{\|\omega\|^{2}}
$$

in which $\delta_{t}$ is the free variable along the twist axis. The equation of the pitch gives rise to two special cases if 1) $\lambda_{t}=0$ or if 2) $\lambda_{t}=\infty$. The first occurs when $\omega$ and $\mathbf{v}$ are perpendicular. This is interpreted as a pure rotation. The latter happens when $\omega=0$ and $\mathbf{v} \neq 0$ and is seen as a pure translation. Later in the balancing process, these special cases require special attention.

Similarly, the linear and angular momentum - respectively denoted with $\mathbf{p}$, and $\boldsymbol{\xi}$-form a screw, the 'momentum' wrench Fig. 1

$$
\mathbf{h}=\left[\begin{array}{l}
\boldsymbol{\xi} \\
\mathbf{p}
\end{array}\right] \quad \boldsymbol{\xi}=\mathbf{r}_{h} \times \mathbf{p}+\lambda_{h} \mathbf{p}
$$

This momentum wrench $\mathbf{h}$ can be interpreted as a linear momentum in the direction of $\mathbf{p}$, passing through point $\mathbf{r}_{h}$ and an angular momentum around this axis, here called the momentum pitch $\lambda_{h}$

$$
\mathbf{r}_{h}=\frac{\mathbf{p} \times \boldsymbol{\xi}}{\|\mathbf{p}\|^{2}}+\delta_{h} \mathbf{p} \quad \lambda_{h}=\frac{\boldsymbol{\xi}^{\top} \mathbf{p}}{\|\mathbf{p}\|^{2}}
$$

in which $\delta_{h}$ is the free variable along the wrench axis. It should be noted that the twist and wrench are dual properties. The screw axis of the twist is formed by its 'rotative' component, the angular velocity. Conversely, for the wrench its 'translative' part, the linear momentum or force, determines the screw axis. Therefore, a wrench is often denoted as a co-screw. Two special cases occur on the momentum wrench; if 1$)$ the linear and angular momentum are perpendicular $\left(\lambda_{h}=0\right)$ or if 2$)$ the momentum wrench is a pure angular momentum $\left(\lambda_{h}=\infty\right)$.

The momentum wrench is formed by the product of a body's mass matrix $\mathbf{M}$ and its twist

$$
\mathbf{h}=\mathbf{M t}
$$


The body's mass matrix is formed by the mass integral over all points $\mathbf{r}$ in the bodies volume $V$. This gives rise to a matrix build up with $m$ as the body's mass, $\mathbf{c}$ the location of the COM and $\mathbf{G}$ the angular inertia matrix expressed around the COM

$$
\begin{aligned}
\mathbf{M} & =\int_{V}\left[\begin{array}{cc}
-[\mathbf{r} \times]^{2} & {[\mathbf{r} \times]} \\
-[\mathbf{r} \times] & \mathbf{I}_{3}
\end{array}\right] \mathrm{d} m \\
& =\left[\begin{array}{cc}
\mathbf{G}-m[\mathbf{c} \times]^{2} & m[\mathbf{c} \times] \\
-m[\mathbf{c} \times] & m \mathbf{I}_{3}
\end{array}\right]
\end{aligned}
$$

In here $[\mathbf{a} \times]$ denotes the skew symmetric matrix of a 3-vector. The angular inertia matrix $\mathbf{G}$ is parameterized by three moments of inertia $g_{x x}, g_{y z}$, and $g_{z z}$, and three products of inertia $g_{x y}, g_{x z}$, and $g_{y z}$. Altogether, the body's mass matrix is determined by 10 inertial parameters. The angular inertia matrix can be diagonalized $\mathbf{G}^{\mathrm{p}}=\operatorname{diag}(\mathbf{g})$ by $\operatorname{rotating}$ it along its principal axes. Here $\mathbf{g}=\left[g_{1}, g_{2}, g_{3}\right]$ denotes the vector of principal moments of inertia

$$
\mathbf{G}=\left[\begin{array}{lll}
g_{x x} & g_{x y} & g_{x z} \\
g_{x y} & g_{y y} & g_{y z} \\
g_{x z} & g_{y z} & g_{z z}
\end{array}\right]=\mathbf{R}^{\top} \mathbf{G}^{\mathrm{p}} \mathbf{R}
$$

This rotation matrix $\mathbf{R}$ can be found by eigenvalue decomposition. It should be noted eight orientations result in a diagonal inertia matrix, i.e. for each octant one. For simplicity sake we choose a sorted inertia vector. From the integral form of the mass matrix (Eq. (6) and the positivity of mass, it can be deducted that the principal moments of inertia must be non-negative, and form a triangle inequality. Therefore we get the following set of inequality conditions on the mass and inertia of a body

$$
\begin{aligned}
m & \geq 0 \\
\mathbf{g} & \geq 0 \\
g_{2}+g_{3} & \geq g_{1} \\
g_{1} \geq g_{2} & \geq g_{3}
\end{aligned}
$$

The lower limits on in these conditions are formed by theoretical objects such as infinitely slender, flat, or perfectly axisymmetric objects.

\subsection{Dynamic balance conditions in screw theory}

Dynamic balance is obtained when the sum of the momentum wrenches of all $n_{\mathrm{b}}$ bodies in the mechanism equate to zero for all motions. In the current notation this reads

$$
\sum_{i=1}^{n_{\mathrm{b}}} \mathbf{h}_{i}=\sum_{i=1}^{n_{\mathrm{b}}} \mathbf{M}_{i} \mathbf{t}_{i}=0
$$

For a closed-loop mechanism the body twists $\left(\mathbf{t}_{i}\right)$ are not independent as they are related through the differential loopclosure constraints. For each of the $n_{\mathrm{d}}$ degrees of freedom (DOF) of the mechanism, a unique twist is associated to each body $\hat{\mathbf{t}}_{i, j}$. This unit twist (denoted with a hat) is defined as the twist of body $i$ generated by a unit velocity actuation of DOF $j$. The actual body twist $\left(\mathbf{t}_{i}\right)$ is formed by the weighted sum over the input velocities $\dot{q}_{j}$ of these unit twists

$$
\mathbf{t}_{i}=\sum_{j=1}^{n_{\mathrm{d}}} \hat{\mathbf{t}}_{i, j} \dot{q}_{j}
$$

Substituting this back into the balancing condition of Eq. (13), we can find the dynamic balancing conditions for each of the $n_{\mathrm{d}}$ DOFs

$$
\sum_{i=1}^{n_{\mathrm{b}}} \hat{\mathbf{h}}_{i, j}=\sum_{i=1}^{n_{\mathrm{b}}} \mathbf{M}_{i} \hat{\mathbf{t}}_{i, j}=0
$$


in which $\hat{\mathbf{h}}_{i, j}$ is the unit momentum wrench of body $i$ as a consequence of unit velocity of DOF $j$. Combination of these balance conditions using Eq. (14) provides a condition on the momentum wrench basis $\left(\mathbf{M}_{\mathrm{B}}\right)$

$$
\mathbf{h}=\left[\begin{array}{lll}
\sum_{i=1}^{n_{\mathrm{b}}} \hat{\mathbf{h}}_{i, 1} & \cdots & \sum_{i=1}^{n_{\mathrm{b}}} \hat{\mathbf{h}}_{1, n_{d}}
\end{array}\right] \dot{\mathbf{q}}=\mathbf{M}_{\mathrm{B}} \dot{\mathbf{q}}=0
$$

Since this should hold for all possible joint velocity, $6 \times n_{\mathrm{d}}$ instantaneous dynamic balance conditions are obtained for the spatial case and $3 \times n_{\mathrm{d}}$ for the planar case.

$$
\mathbf{M}_{\mathrm{B}}=0
$$

In the remainder of this section these equations are solved for the inertial parameters in the mass matrix to yield dynamic balance in the pose of inspection.

\subsection{Approach to solve dynamic balance conditions}

The instantaneous dynamic balance method presented here consists of two steps. In step A, the dynamic balance conditions $($ Eq. (15) are solved on mechanism level by requiring a specific relation between the twist and momentum wrench for $n_{\mathrm{d}}$ bodies. In step $\mathrm{B}$, this twist-momentum relation is solved to find the corresponding mass matrix of these $n_{\mathrm{d}}$ bodies. In both steps, special cases are identified and treated. In order ensure that feasible inertia parameters Eq. (9) - Eq. (11) are found in step B, a detailed description of the attainable momentum (momentum span) a function the inertial parameters and twist is given. Therefore, in the next paragraphs the description of the two steps is interleaved with a screw based interpretation of the momentum span.

\subsection{Step A. Dynamic balance solution on mechanism level}

The $6 \times n_{\mathrm{d}}$ dynamic balancing conditions $($ Eq. (15) $)$ are solved by uniquely associating a momentum wrench and a twist to $n_{\mathrm{d}}$ mass matrices. This is done by splitting the mechanism in a proximal and distal set of bodies and joints. The first set is a collection of $n_{\mathrm{d}}$ joints and bodies closest to base. These joints will function as the input coordinates or DOFs of the mechanism. The second set are the remaining 'distal' bodies and dependent joints. The inertial parameters of the $n_{\mathrm{d}}$ proximal bodies, directly connected to the input joints, will be determined as a function of the inertial parameters of the distal part. In this hierarchical balancing procedure the total momentum wrench generated by actuation of the most distal input joints is determined first. For this motion to be dynamically balanced, the momentum generated by the body connected to this input joint $(j)$ should be equal and opposite to the momentum generated by the distal part of the mechanism

$$
-\sum_{i \neq j} \hat{\mathbf{h}}_{i, j}=\hat{\mathbf{h}}_{j, j}=\mathbf{M}_{j} \hat{\mathbf{t}}_{j, j}
$$

As the proximal input joints and connected bodies are kept fixed, only the inertial parameters of the distal bodies show up in the left side of this equation. The inertial parameters $\left(\mathbf{M}_{j}\right)$ for this input body can be computed via equations which are presented later. After this first mass matrix is solved, the approach is repeated for the second most distal link and so on, until all inertial parameters of the $n_{\mathrm{d}}$ proximal bodies are determined. As the choice of input joints is not unique, a variety of sequences can be used as long as no passive joints are in between the input joints and the base. Also, it is required that the chosen parameterization does not result in a singular configuration of the mechanism.

\subsection{Intermezzo: Screw theory interpretation of momentum span}

Before the mass matrices $\mathbf{M}_{j}$ are extracted from Eq. (18), we need to know which momentum wrenches are admissible for any twist-mass matrix combination. Later on this relation is inverted to find mass matrix for a given momentum wrench and twist. Moreover, we wish to know how to ensure that feasibility bounds (Eq. (9)-Eq. (11)] are respected by the solution. Furthermore, special cases need to be identified to see if additional conditions are required. In the first part, the twist-momentum relation is interpreted in a screw theory manner, following the work of Selig et al. [31]. In the second part, a detailed study of the angular momentum span is conducted to ensure the feasibility bounds on the moments of inertia. 
Using Eq. (3) and Eq. (7) the expression for the linear and angular momentum becomes

$$
\begin{aligned}
\mathbf{p} & =-m \mathbf{c} \times \boldsymbol{\omega}+m \mathbf{v} \\
& =m\left(\mathbf{r}_{t}-\mathbf{c}\right) \times \boldsymbol{\omega}+m \lambda_{t} \boldsymbol{\omega} \\
\boldsymbol{\xi} & =\mathbf{G} \boldsymbol{\omega}+\mathbf{c} \times \mathbf{p}
\end{aligned}
$$

Following the interpretation of [31], a plane is constructed through the twist $\mathbf{t}$ and the COM c, the t-c-plane (Fig. 1]. The point where the momentum wrench passes through this $\mathbf{t}$-c-plane is termed $\mathbf{r}_{h, \mathrm{~s}}$. In their paper, following the work of Ball [25], Selig et al. show that this point is simultaneously the shortest distance point between $\mathbf{t}$ and $\mathbf{h}$. The corresponding shortest distance point on the twist axis is $\mathbf{r}_{t, \mathrm{~s}}$. Without any moment of inertia, i.e. when the body is a point mass, the momentum wrench passes through c. With increased angular momentum the line moves further away from $\mathbf{t}$ and $\mathbf{c}$. The exact extent of the angular momentum span will be studied later in this section. The linear momentum vector is not normal to the t-c-plane due to the influence of the twist pitch (Eq. (20)].

In the general finite twist pitch case, the momentum pitch is found to be

$$
\lambda_{h}=\frac{\boldsymbol{\xi}^{\top} \mathbf{p}}{\|\mathbf{p}\|^{2}}=\frac{\lambda_{t} \omega^{\top} \mathbf{G} \omega+\omega^{\top}\left[\mathbf{c}-\mathbf{r}_{t} \times\right] \mathbf{G} \boldsymbol{\omega}}{m\|\omega\|^{2}\left(\lambda_{t}^{2}+l_{\mathrm{c}}^{2}\right)}
$$

in here $l_{\mathrm{c}}$ is the distance between the COM and the twist axis.

\subsubsection{Special cases}

Special cases for the momentum occur when 1) the momentum pitch is zero, or 2) when the momentum pitch is infinite. The combination of twist and the mass of the body for which these special conditions occur are given here. We exclude trivial cases such as $m=0$.

1. The zero pitch momentum $\left(\lambda_{h}=0\right)$ occurs when $\mathbf{p} \perp \boldsymbol{\xi}$. The momentum pitch (Eq. (22) is zero when the twist pitch has a specific relation with the angular velocity

$$
\lambda_{t}=-\frac{\omega^{\top}\left[\mathbf{c}-\mathbf{r}_{t} \times\right] \mathbf{G} \boldsymbol{\omega}}{\omega^{\top} \mathbf{G} \omega}
$$

Furthermore, when the twist pitch is infinite $\left(\lambda_{t}=\infty\right)$, Eq. (21) and Eq. (19) reduce to

$$
\mathbf{p}=m \mathbf{v}
$$

such that in $\mathbf{p} \perp \boldsymbol{\xi}$, resulting in a zero momentum pitch.

2. An infinite pitch momentum $\left(\lambda_{h}=\infty\right)$ appears when $\|\mathbf{p}\|=0$. From Eq. (20) it is deduced that this is only the case when the body is in pure rotation $\left(\lambda_{t}=0\right)$ around the $\operatorname{COM}\left(l_{c}=0\right)$.

This means that an infinite pitch twist will always result in a zero pitch momentum and furthermore, an infinite pitch momentum can only be generated by a zero pitch twist. Refer to Table 1 for an overview of the conditions.

\subsubsection{Angular momentum span}

The angular momentum span is required to completely define the momentum limits for a given body. For brevity reasons, we group the influence of the $\mathrm{COM}$ on the angular momentum

$$
\boldsymbol{\xi}_{c}=\boldsymbol{\xi}-\mathbf{c} \times \mathbf{p}=\mathbf{G} \omega
$$

Now, we are interested to see what values for $\boldsymbol{\xi}_{c}$ can be obtained for any given angular velocity, principal moments of inertia and orientations of the body

$$
\boldsymbol{\xi}_{c}=\mathbf{R}^{\top} \mathbf{G}^{\mathrm{p}} \mathbf{R} \omega
$$

Without loss of generality we choose $\omega$ along the global $z$-axis and $\mathbf{R}$ with three consecutive rotations according the $z-x-z$ convention

$$
\mathbf{R}=\mathbf{R}_{z}\left(\phi_{1}\right) \mathbf{R}_{x}\left(\phi_{2}\right) \mathbf{R}_{z}\left(\phi_{3}\right)
$$


Since $\omega$ is invariant for $\mathbf{R}_{z}$ we have for the angular momentum the following product of matrices

$$
\boldsymbol{\xi}_{c}=\mathbf{R}_{z}^{\top}\left(\phi_{3}\right) \mathbf{R}_{x}^{\top}\left(\phi_{2}\right) \mathbf{R}_{z}^{\top}\left(\phi_{1}\right) \mathbf{G}^{\mathrm{p}} \mathbf{R}_{z}\left(\phi_{1}\right) \mathbf{R}_{x}\left(\phi_{2}\right) \boldsymbol{\omega}
$$

Which after expansion this becomes:

$$
\boldsymbol{\xi}_{c}=g_{3} \omega+\sin \left(\phi_{2}\right) \mathbf{R}_{z}^{\top}\left(\phi_{3}\right) \mathbf{R}_{x}^{\top}\left(\phi_{2}+\frac{\pi}{2}\right) \cdot\left(\left(g_{2}-g_{3}+\frac{g_{1}-g_{2}}{2}\right) \omega+\frac{g_{1}-g_{2}}{2} \mathbf{R}_{y}\left(2 \phi_{1}\right) \omega\right)
$$

The effect of the angles is on the angular momentum depicted in Fig. 2. From the previous equation it follows that angular momentum $\boldsymbol{\xi}_{c}$ lies on circle - parameterized by $2 \phi_{1}-$ which, in turn, lies on a plane passing through $g_{3} \omega$. The angle of the plane with the $y$-axis is given by $\phi_{2}$. The size and center of the circle scale with $\sin \left(\phi_{2}\right)$. This whole figure is rotated by $\phi_{3}$ around the $\mathbf{n}_{z}$-axis. This means that, for all orientations, the angular momentum $\boldsymbol{\xi}_{c}$ will be contained inside a sphere with two spherical cavities. These three spheres touch at $g_{1} \omega, g_{2} \omega$, and $g_{3} \omega$. From this interpretation, three bounds on $\boldsymbol{\xi}_{c}$ can be deducted, which are respected for any orientation of the body

$$
\begin{aligned}
\left\|\boldsymbol{\xi}_{c}-1 / 2\left(g_{1}+g_{3}\right) \boldsymbol{\omega}\right\| & \leq 1 / 2\left(g_{1}-g_{3}\right) \\
\left\|\boldsymbol{\xi}_{c}-1 / 2\left(g_{1}+g_{2}\right) \boldsymbol{\omega}\right\| & \geq 1 / 2\left(g_{1}-g_{2}\right) \\
\left\|\boldsymbol{\xi}_{c}-1 / 2\left(g_{2}+g_{3}\right) \boldsymbol{\omega}\right\| & \geq 1 / 2\left(g_{2}-g_{3}\right)
\end{aligned}
$$

This also gives a critical maximal value for the principal moments of inertia, such that a given $\boldsymbol{\xi}_{c}$ lies on the surface of one of the spheres. The cosine law yields the maximal moment of inertia:

$$
\begin{aligned}
& g_{1} \leq g_{1, \text { crit }}=\frac{\boldsymbol{\xi}_{c}^{\top}\left(g_{2} \omega-\boldsymbol{\xi}_{c}\right)}{\boldsymbol{\omega}^{\top}\left(g_{2} \omega-\boldsymbol{\xi}_{c}\right)} \\
& g_{2} \leq g_{2, \text { crit }}=\frac{\boldsymbol{\xi}_{c}^{\top}\left(g_{3} \omega-\boldsymbol{\xi}_{c}\right)}{\boldsymbol{\omega}^{\top}\left(g_{3} \omega-\boldsymbol{\xi}_{c}\right)} \\
& g_{3} \leq g_{3, \text { crit }}=\frac{\boldsymbol{\xi}_{c}^{\top}\left(g_{1} \omega-\boldsymbol{\xi}_{c}\right)}{\omega^{\top}\left(g_{1} \omega-\boldsymbol{\xi}_{c}\right)}
\end{aligned}
$$

When the principal moments of inertia are chosen equal, i.e. $g_{1}=g_{2}$ or $g_{2}=g_{3}$, the angular momentum span collapses to the surface of a sphere. In case all principal moments of inertia are equal, i.e. $g_{1}=g_{2}=g_{3}$, the angular momentum span collapses to a point on the $\omega$-axis.

Since the principal moments of inertia are all positive, for all bodies and orientations the absolute angle between the momentum and the angular velocity $(\theta)$ will be smaller than $\pi / 2$, therefore

$$
\omega^{\top} \boldsymbol{\xi}_{c}=\|\omega\|\left\|\boldsymbol{\xi}_{c}\right\| \cos (\theta)>0
$$

\subsection{Step B. Dynamic balance solution on body level}

Now that we have a geometric interpretation of the momentum span, we will extract the inertial parameters from the $6 \times n_{\mathrm{d}}$ instantaneous dynamic balance condition of Eq. (18). For each of the $n_{\mathrm{d}}$ bodies we have to satisfy 6 dynamic balance conditions, for which we can use 10 parameters. This means that in general we can choose 4 parameters (within bounds) and solve for the remaining 6 parameters. We will do this in three steps. First, the force balance conditions are solved. Second, the moment balance solution are obtained. Third, the special cases such as the planar case are discussed.

\subsubsection{Force balance}

In connection with Eq. (18) the force balance is found by extracting the mass and COM fromEq. (19)

$$
m=\frac{1}{\lambda_{t}} \frac{\omega^{\top} \mathbf{p}}{\|\omega\|^{2}}, \quad \mathbf{c}=-\frac{[\omega \times](m \mathbf{v}-\mathbf{p})}{m\|\omega\|^{2}}+\alpha \omega
$$

For brevity reasons the subscripts of Eq. (18) are omitted. It can be seen that mass is completely fixed and the COM must lie on a line parametrized by a variable $\alpha$ allong $\omega$. For a positive mass $(m>0)$ it is required that $\omega^{\top} \mathbf{p}$ and $\lambda_{t}$ have the same sign. 


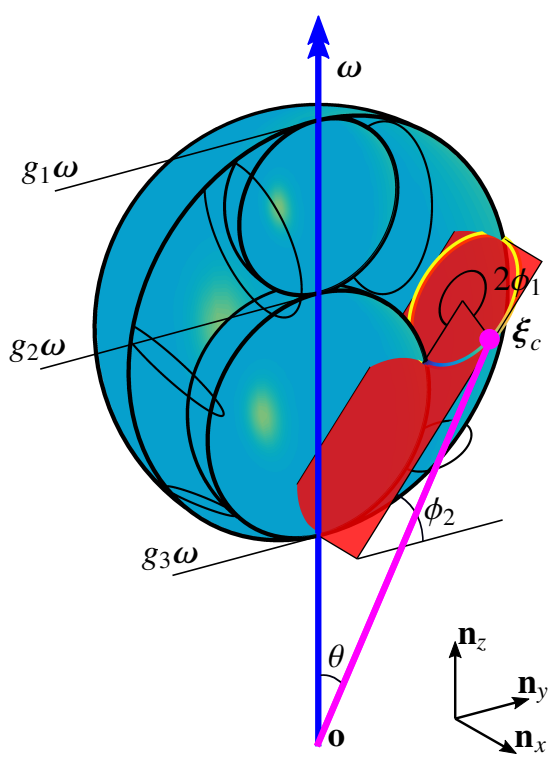

Figure 2: For all orientations of a body, the angular momentum $\xi_{c}$ generated by angular velocity $\omega$ will lie inside a sphere with two spherical cavities, of which the dimensions and position are determined by the bodies principal moments of inertia $g_{1}, g_{2}$, and $g_{3}$. Here the orientation of the body is parameterized by $\phi_{1}, \phi_{2}$, and $\phi_{3}$ (currently $\phi_{3}=0$ ) around local $\mathbf{n}_{z}, \mathbf{n}_{x}$, and $\mathbf{n}_{z}$ axes.

\subsubsection{Moment balance}

Moment balance imposes three conditions Eq. (25) with 6 inertial parameters to be determined. We solve this undetermined set of equations by selecting the principal moments of inertia (g) and solving for the orientation of the body ( $\mathbf{R}$ in Eq. (8)). An equally valid approach would be to choose an orientation and then solve for the principal moments of inertia. However, in that case the incorporation of the feasibility constraints Eq. (10) and Eq. (11) is much harder. It should be noted that $\xi_{c}$ depends on $\mathbf{c}$ and therefore on the choice of $\alpha$.

To solve the moment balance, firstly a local frame will be aligned with the principal axis of inertia. Secondly, the angular velocity as expressed in this local frame $\left(\omega^{\mathrm{p}}\right)$ will be determined. Thirdly, the rotation matrix - mapping the global to the local angular velocity $\omega^{\mathrm{p}}=\mathbf{R} \omega$ - will be extracted.

For the local angular velocity vector there hold three conditions

$$
\begin{aligned}
\|\omega\|^{2} & =\left\|\omega^{\mathrm{p}}\right\|^{2} \\
\omega^{\top} \boldsymbol{\xi}_{c} & =\left(\omega^{\mathrm{p}}\right)^{\top} \mathbf{G}^{\mathrm{p}} \boldsymbol{\omega}^{\mathrm{p}} \\
\left\|\boldsymbol{\xi}_{c}\right\|^{2} & =\left(\omega^{\mathrm{p}}\right)^{\top}\left(\mathbf{G}^{\mathrm{p}}\right)^{2} \boldsymbol{\omega}^{\mathrm{p}}
\end{aligned}
$$

This can be rewritten as matrix-vector relation on the element-wise quadratic of the local angular velocity denoted with $\left(\omega^{\mathrm{p}}\right)^{\circ 2}$

$$
\left[\begin{array}{l}
\|\omega\|^{2} \\
\omega^{\top} \boldsymbol{\xi}_{c} \\
\left\|\boldsymbol{\xi}_{c}\right\|^{2}
\end{array}\right]=\left[\begin{array}{lll}
\mathbf{1} & \mathbf{g} & \mathbf{g}^{\circ 2}
\end{array}\right]^{\top}\left(\omega^{\mathrm{p}}\right)^{\circ 2}
$$

which leads to a solution of the local angular velocity vector as

$$
\left(\omega^{\mathrm{p}}\right)^{\circ 2}=\left[\begin{array}{lll}
\mathbf{1} & \mathbf{g} & \mathbf{g}^{\circ 2}
\end{array}\right]^{-\top}\left[\begin{array}{l}
\|\omega\|^{2} \\
\omega^{\top} \boldsymbol{\xi}_{c} \\
\left\|\boldsymbol{\xi}_{c}\right\|^{2}
\end{array}\right]
$$




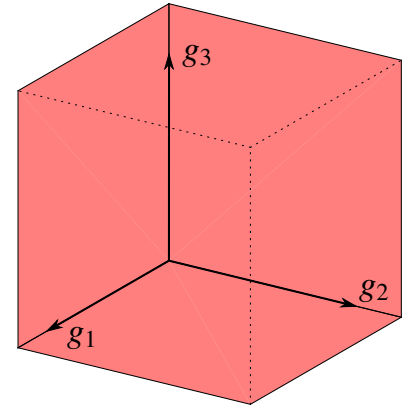

(a)

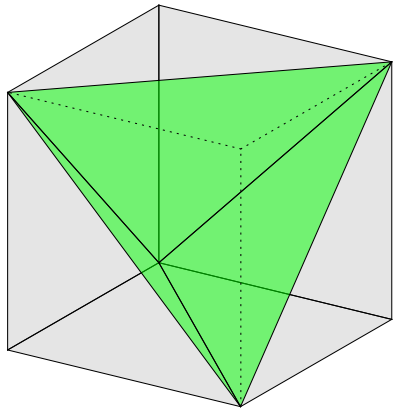

(b)

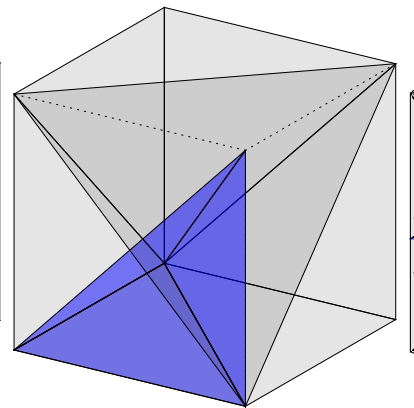

(c)

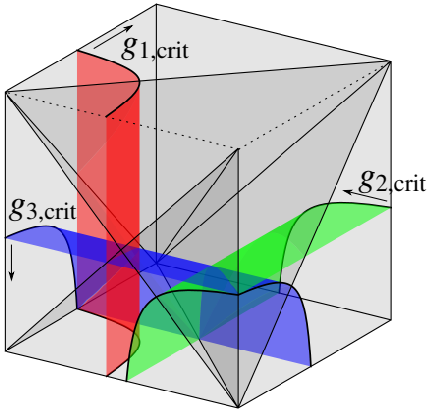

(d)

Figure 3: The four types of bounds on the selection of the principal moments of inertia in the balancing process. (a) positive principal moments of inertia $\mathbf{g}>0$, (b) The triangle inequality $g_{i} \leq g_{j}+g_{k}$, (c) the sorting condition $g_{1} \geq g_{2} \geq g_{3}$ (d) critical principal moments of inertia Eq. (33)Eq. (33) for a given $\omega$ and $\boldsymbol{\xi}_{c}$

This matrix inversion can be done as long as all principal moments of inertia are different $g_{1} \neq g_{2} \neq g_{3}$. In the next section, equal principal moments of inertia will be investigated. Eight distinct solutions for $\omega^{\mathrm{p}}=\operatorname{diag}([ \pm 1, \pm 1, \pm 1]) \sqrt{\left(\omega^{\mathrm{p}}\right)^{\circ 2}}$ are found, each corresponding to an octant. The positivity conditions $\left(\omega^{\mathrm{p}}\right)^{\circ 2}>0$ are satisfied as long as $\mathbf{g}$ is chosen such that the angular momentum limits Eq. (30)-Eq. (32) are satisfied.

The rotation matrix $(\mathbf{R})$ can be found between the local and global angular velocity vector by recognizing that the following system of equations must hold

$$
\begin{aligned}
\mathbf{R}\left[\begin{array}{lll}
\omega & \boldsymbol{\xi}_{c} & \boldsymbol{\omega} \times \boldsymbol{\xi}_{c}
\end{array}\right] & =\left[\begin{array}{lll}
\omega^{\mathrm{p}} & \mathbf{G}^{\mathrm{p}} \boldsymbol{\omega}^{\mathrm{p}} & \boldsymbol{\omega}_{l} \times \mathbf{G}^{\mathrm{p}} \boldsymbol{\omega}^{\mathrm{p}}
\end{array}\right] \\
\mathbf{R A} & =\mathbf{B}
\end{aligned}
$$

which can be inverted if $\omega$ and $\xi_{c}$ are not co-linear, yielding

$$
\mathbf{R}=\mathbf{B} \mathbf{A}^{-1}
$$

In this approach the principal moments of inertias are free to choose. However, we saw that for the resulting $\left(\omega^{\mathrm{p}}\right)^{\circ 2}$ to be positive, $\mathrm{g}$ must be selected such that Eq. (30)-Eq. (32) are respected. Furthermore, also the positive definiteness of the moment of inertia matrix (Eq. (10) must be respected in addition to the triangle inequality Eq. (11) and the sorting condition Eq. (12) These constraints can easily be incorporated by limiting the choice of $\mathbf{g}$. Giving a total set of 9 limiting conditions of the choice of $\mathbf{g}$. In Fig. 3 a graphical interpretation of these conditions can found.

Now to summarize, feasible instantaneous dynamic balance solutions can be found as long as 1) the twist pitch $\lambda_{t}$ and $\omega^{\top} \mathbf{p}$ have the same sign, and 2) $\omega^{\top} \boldsymbol{\xi}_{c}>0$. In genera,l there are four parameters to choose within bounds: $\alpha$ on the $\mathrm{COM}$ and $\mathbf{g}$ on the moment of inertia matrix.

\subsubsection{Special conditions}

In several special conditions this general solution requires additional conditions. Firstly, when the principal moments of inertia are chosen equal and the inverse in Eq. (42) does not exist. Secondly, when $\boldsymbol{\omega}$ and $\boldsymbol{\xi}_{c}$ are collinear and the inverse in Eq. (45) does not exist. Thirdly, when the pitches of the twist or momentum wrench are either zero or infinite. Fourthly and lastly, in the planar case the out of plane components can be ignored leading to a reduced solution space.

1) In case an axisymmetric body is chosen such that $g_{1}=g_{2}$ or $g_{2}=g_{3}$ the solution to $\omega^{\mathrm{p}}$ in Eq. (42) gains a freedom, allowing for different orientations of the body without changing the corresponding moment of inertia matrix. In that case, the values of the equal $\left(g_{2}=g_{\mathrm{e}}\right)$ principal moments of inertia cannot be chosen freely as $\boldsymbol{\xi}_{c}$ should lie on the surface of a sphere Eq. (33) -Eq. (35) and therewith determining the remaining moment of inertia $\left(g_{\mathrm{u}}\right)$

$$
g_{2}=g_{\mathrm{e}}=\frac{\boldsymbol{\xi}_{c}^{\top}\left(g_{\mathrm{u}} \omega-\boldsymbol{\xi}_{c}\right)}{\omega^{\top}\left(g_{\mathrm{u}} \omega-\boldsymbol{\xi}_{c}\right)}
$$


Table 1: For several special cases the general conditions (G.C.) of Eq. (37) and Eq. (45) do not hold. Either the combination of twist and wrench are not possible (N.P.) or require additional conditions. Additional parameter freedom is denoted with a $i$.

\begin{tabular}{l|cccc} 
& $\left|\lambda_{t}\right|=(0, \infty)$ & \multicolumn{2}{c}{$\left|\lambda_{t}\right|=0$} & $\left|\lambda_{t}\right|=\infty$ \\
\hline$\left|\lambda_{h}\right|=(0, \infty)$ & G.C. & $\omega \perp \mathbf{p}, \quad m=i$ & N.P. \\
$\left|\lambda_{h}\right|=0$ & G.C. & $\omega \perp \mathbf{p}$ & $m=i$ & $\mathbf{v} \| \mathbf{p}, \quad \mathbf{G}=i$ \\
$\left|\lambda_{h}\right|=\infty$ & N.P. & $\mathbf{p}=0, \quad m=i$ & N.P.
\end{tabular}

Equal principal moments of inertia $g_{1}=g_{2}=g_{3}$ can only be chosen if $\boldsymbol{\xi}_{c}$ and $\omega$ are co-linear, then $\mathbf{R}$ is a free choice that does not change the inertia matrix. For the $g_{i}$ 's the following condition must hold

$$
g_{1}=g_{2}=g_{3}=\frac{\left\|\boldsymbol{\xi}_{c}\right\|}{\|\boldsymbol{\omega}\|}
$$

2) In general, when $\xi_{c}$ and $\omega$ are co-linear, both should be aligned along one of the principal axis, fixing only the corresponding principal moment of inertia. The other principal moments of inertia and the orientation around that principal axis are free to choose.

3) In previous sections several special cases of the twist and momentum wrench are identified. For the dynamic balance some of these require special attention. We have seen that both the twist and the required momentum wrench can be finite, infinite or zero pitched (co-) screws. This leads to 9 cases as shown in table Table 1. Based on twist pitch and momentum pitch equations, respectively Eq. (2) and Eq. (22), we discuss 3 conditions, from which the whole table can be deducted.

- In the limit case $\lambda_{t}=0$, the body is in pure rotation. Therefore $\omega$ and $\mathbf{p}$ should be perpendicular. This leaves $m$ in Eq. (37) indeterminate and free to choose.

- In the limit case $\lambda_{t}=\infty$ the body is in pure translation $(\omega=0)$. Therefore, this requires $\mathbf{v} \| \mathbf{p}$. This bounds $\mathbf{c}$ to line due to $\boldsymbol{\xi}=\mathbf{c} \times \mathbf{p}$ in Eq. (21) The balancing equations become

$$
m=\frac{\|\mathbf{p}\|}{\|\mathbf{v}\|} \quad \mathbf{c}=\frac{\mathbf{p} \times \boldsymbol{\xi}}{\|\mathbf{p}\|^{2}}+\alpha \mathbf{p}
$$

Therefore $\boldsymbol{\xi}$ and $\mathbf{p}$ should be perpendicular, requiring $\lambda_{h}=0$. Other values for $\lambda_{h}$ are not possible. Therefore, $\mathbf{G}$ is free to choose.

- The limit case $\lambda_{h}=\infty$ occurs only when $\mathbf{p}=0$. This places $\mathbf{c}=\mathbf{r}_{t}+\alpha \omega$ on the twist axis. Therefore, it requires $\lambda_{t}=0$. Other values for $\lambda_{t}$ are not possible in this case.

4) In the planar case we can ignore the out of plane parts and we are left with a single inertia value $g$. We have two cases either $\lambda_{t}=0$, in which $m$ is indeterminate, or $\lambda_{t}=\infty$, in which $g$ is indeterminate. In the first case Eq. (20) and Eq. (21) are solved by

$$
\begin{aligned}
& \mathbf{c}=\mathbf{r}_{t}-\frac{1}{m} \frac{\omega \times \mathbf{p}}{\|\omega\|^{2}}, \\
& g=\frac{\omega^{\top}\left(\boldsymbol{\xi}-\mathbf{r}_{t} \times \mathbf{p}\right)}{\|\omega\|^{2}}-\frac{1}{m} \frac{\|\mathbf{p}\|^{2}}{\|\omega\|^{2}}
\end{aligned}
$$

In the second case $\lambda_{t}=\infty$ the body is in pure translation and we require $\mathbf{v} \| \mathbf{p}$, refer to Table 1 . Therefore, similar to the spatial case $m$ and $\mathbf{c}$ are determined according to Eq. (48) and $g$ is undetermined.

All these special conditions impose additional requirements on $\mathbf{t}$ and $\mathbf{h}$. When following the hierarchical dynamic balancing procedure, as presented in this paper, these additional constraints are inherited over to the bodies higher in the chain, limiting their choice of mass distribution. 


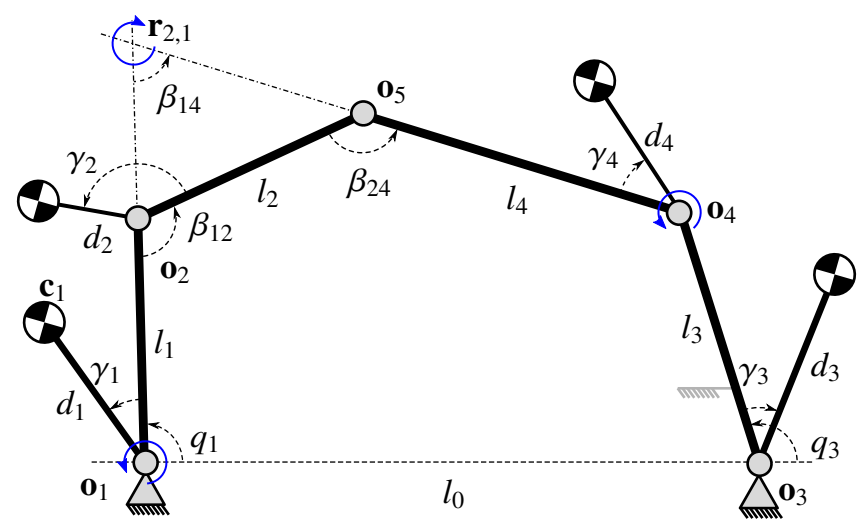

Figure 4: The kinematics of the 5R planar five-bar mechanism. The solid arrows indicate axis of rotation of moving bodies due to actuation of $q_{1}$ and fixation of $q_{3}$. For body 2 , the axis of rotation lies at $\mathbf{r}_{2,1}$.

\subsubsection{Global dynamic balance}

With this a dynamic balancing procedure is available, which yields instantaneous dynamic balance for any type of mechanisms as long as the differential kinematics are known. As discussed earlier this instantaneous property only hold in the pose of inspection. Outside this particular pose the dynamic balance is not necessarily maintained. To obtain global dynamic balance, the previously derived dynamic balance solutions should be valid for all possible poses and motions. The homogenization of this step is outside the scope of this paper.

\subsubsection{Reactionless paths}

When a multi-DOF system with sufficient DOFs is partially balanced, for example only the shaking forces are canceled, a subset of the DOF can be used to generate full dynamic balance [21, 22]. If these partially balanced mechanisms are placed in a instantaneously dynamically balanced pose, locally the full mobility can be used. This means that in these poses, multiple reactionless paths meet.

These paths are computed, similar to [22], by choosing the coordinate velocities on the null-space of the momentum basis Eq. (16)

$$
\dot{\mathbf{q}} \in \operatorname{ker}\left(\mathbf{M}_{\mathrm{B}}\right)
$$

By numerically integrating these null-space velocities, dynamically balanced paths are computed. In general, this $\mathbf{M}_{B}$ matrix is full rank and does not possess a null-space, however in the case the system is partially balanced and has more DOF than unbalanced directions, a null-space appears, which can be used to find such reactionless paths. Since conservation of angular momentum is a non-holonomic condition [32], a closed form description of the reactionless paths is in general not possible. In the instantaneously dynamically balanced poses, the momentum basis $\left(\mathbf{M}_{\mathrm{B}}\right)$ is a null matrix, locally allowing for the full mobility to be used. Therefore, in these poses the shaking wrench is not influenced by the joint accelerations.

\section{Design of a dynamically balanced five-bar mechanism}

\subsection{Kinematics and dynamics}

To illustrate the presented instantaneous dynamic balancing methodology, and to show that instantaneous dynamic balance results in intersecting reactionless paths, we apply the method to a 2-DOF planar 5R mechanism. This five-bar mechanism is parameterized as two serial chains with revolute joints at $\mathbf{o}_{1}$ till $\mathbf{o}_{4}$, joined together at the end-effector $\mathbf{0}_{5}$, as shown in Fig. 4 . The distance between the base joints $\mathbf{o}_{1}$ and $\mathbf{o}_{3}$ is length $l_{0}$. The length of the four moving bodies are $l_{1}$ till $l_{4}$. For later use in the differential kinematics, auxiliary angles $\beta_{i j}$ between body $i$ and body $j$ are used. To each body, a mass $m_{i}$, and a moment of inertia $g_{i}$ are associated. The center of mass $\mathbf{c}_{i}$ of each body is defined by a distance $d_{i}$ and an angle $\gamma_{i}$. 


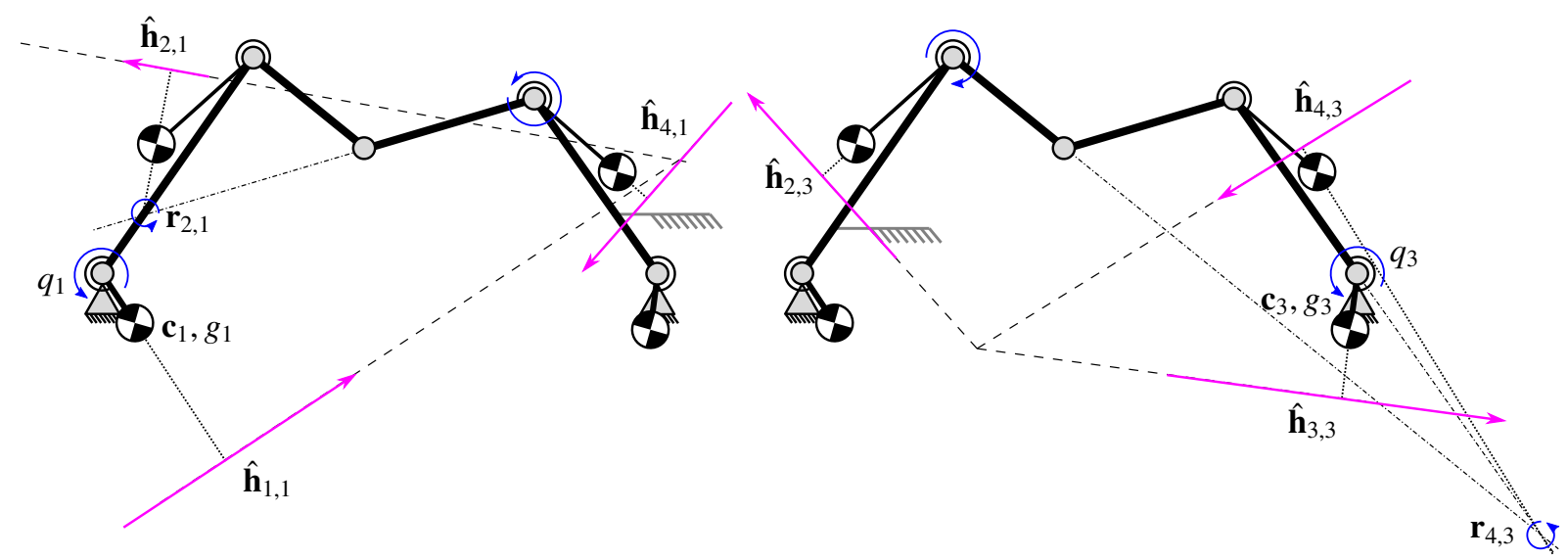

Figure 5: A graphical representation of the instantaneous dynamic balance procedure of a 5R planar five-bar. Left shows that the momentum wrenches of the bodies (straight arrows) due to the actuation of the first DOF (joint $q_{1}$ ) sum to zero. Right shows the zero sum of the momentum wrenches for the second DOF, the actuation of joint $q_{3}$.

For instantaneous dynamic balance, only the differential kinematics are required. The loop closure equations are assumed to be satisfied, and not discussed here. According to the hierarchical balancing procedure, as described in previous sections, the two base joints $q_{1}$ and $q_{3}$ are chosen as the input joints. For each of the DOFs the mechanism will act as a four-bar mechanism. We will only apply this procedure for the first DOF $\left(q_{1}\right)$. The second DOF $\left(q_{3}\right)$ can be treated similarly. The angular velocity of the input link is the unit vector perpendicular to the plane of the robot $\hat{\omega}_{1,1}=\mathbf{n}_{z}$. The twist of the bodies due unit velocity of joint 1 become

$$
\begin{array}{rlrl}
\hat{\mathbf{t}}_{1,1} & =\left[\begin{array}{c}
\mathbf{n}_{z} \\
\mathbf{o}_{1} \times \mathbf{n}_{z}
\end{array}\right], & \hat{\mathbf{t}}_{2,1} & =\left[\begin{array}{c}
\hat{\boldsymbol{\omega}}_{2,1} \\
\mathbf{r}_{2,1} \times \hat{\boldsymbol{\omega}}_{2,1}
\end{array}\right], \\
\hat{\mathbf{t}}_{3,1} & =\left[\begin{array}{l}
\mathbf{0} \\
\mathbf{0}
\end{array}\right], & \hat{\mathbf{t}}_{4,1}=\left[\begin{array}{c}
\hat{\boldsymbol{\omega}}_{4,1} \\
\mathbf{o}_{4} \times \hat{\boldsymbol{\omega}}_{4,1}
\end{array}\right]
\end{array}
$$

in which the angular velocities of link 2 and 4 are

$$
\hat{\boldsymbol{\omega}}_{2,1}=\frac{l_{1}}{l_{2}} \frac{\sin \left(\beta_{14}\right)}{\sin \left(\beta_{24}\right)} \mathbf{n}_{z}, \quad \hat{\boldsymbol{\omega}}_{4,1}=\frac{l_{1}}{l_{4}} \frac{\sin \left(\beta_{12}\right)}{\sin \left(\beta_{24}\right)} \mathbf{n}_{z}
$$

The instantaneous center of rotation of body $\mathbf{r}_{2,1}$ is on the intersection of the line through $\mathbf{o}_{1}$ and $\mathbf{o}_{2}$ and the line through $\mathbf{0}_{4}$ and $\mathbf{0}_{5}$

$$
\mathbf{r}_{2,1}=\mathbf{o}_{2}+\frac{l_{4} \sin \left(\beta_{12}\right)}{l_{1} \sin \left(\beta_{14}\right)}\left(\mathbf{o}_{1}-\mathbf{o}_{2}\right)
$$

Similar conditions can be derived for the second DOF, the rotation of base joint 3 , yielding $\hat{\mathbf{t}}_{2,3}, \hat{\mathbf{t}}_{3,3}$, and $\hat{\mathbf{t}}_{4,3}$.

\subsection{Dynamic balance conditions of a five-bar}

Instantaneous dynamic balance is obtained by inspecting the two DOFs of the mechanism independently. In Fig. 5 this procedure is depicted graphically. For instantaneous dynamic balance of the first DOF, the momentum of the two distal bodies 2 and 4 should be equated to that of the proximal body 1

$$
\hat{\mathbf{h}}_{1,1}=-\hat{\mathbf{h}}_{2,1}-\hat{\mathbf{h}}_{4,1}
$$

Such that the linear and angular momentum generated by body 1 becomes

$$
\begin{aligned}
& \hat{\mathbf{p}}_{1,1}=-\hat{\mathbf{p}}_{2,1}-\hat{\mathbf{p}}_{4,1} \\
& \hat{\boldsymbol{\xi}}_{1,1}=-\hat{\boldsymbol{\xi}}_{2,1}-\hat{\boldsymbol{\xi}}_{4,1}
\end{aligned}
$$


with the linear and angular momentum Eq. (20) and Eq. (21) of the distal bodies as

$$
\begin{aligned}
& \hat{\mathbf{p}}_{2,1}=m_{2}\left(\mathbf{r}_{2,1}-\mathbf{c}_{2}\right) \times \hat{\boldsymbol{\omega}}_{2,1} \\
& \hat{\mathbf{p}}_{4,1}=m_{4}\left(\mathbf{o}_{4}-\mathbf{c}_{4}\right) \times \hat{\boldsymbol{\omega}}_{4,1} \\
& \hat{\boldsymbol{\xi}}_{2,1}=-g_{2} \hat{\boldsymbol{\omega}}_{2,1}-\mathbf{c}_{2} \times \hat{\mathbf{p}}_{2,1} \\
& \hat{\boldsymbol{\xi}}_{4,1}=-g_{4} \hat{\boldsymbol{\omega}}_{4,1}-\mathbf{c}_{4} \times \hat{\mathbf{p}}_{4,1}
\end{aligned}
$$

This allows us to derive the moment of inertia and COM of base links 1 and 3 as function the inertial parameters of links 2 and 4 and a choice of mass using Eq. (49) and Eq. (50). For link 1 we have

$$
\begin{aligned}
& \mathbf{c}_{1}=\mathbf{o}_{1}+\frac{\mathbf{n}_{z} \times\left(\hat{\mathbf{p}}_{2,1}+\hat{\mathbf{p}}_{4,1}\right)}{m_{1}}, \\
& g_{1}=\mathbf{n}_{z}^{\top}\left(\mathbf{o}_{1} \times\left(\hat{\mathbf{p}}_{2,1}+\hat{\mathbf{p}}_{4,1}\right)-\hat{\boldsymbol{\xi}}_{2,1}-\hat{\boldsymbol{\xi}}_{4,1}\right)-\frac{\left\|\hat{\mathbf{p}}_{2,1}+\hat{\mathbf{p}}_{4,1}\right\|^{2}}{m_{1}}
\end{aligned}
$$

For link 3 similar conditions hold.

This means that the inertial parameters of link 2 and 4 and the masses of link 1 and 3 can be chosen freely as long as the resulting moments of inertia are positive. This positivity is ensured if the required momentum lines $\hat{\mathbf{h}}_{1,1}$ and $\hat{\mathbf{h}}_{3,3}$ pass counter-clock wise around their revolute joints. Already the solutions to six dynamic balance conditions are found; four force balance and two moment balance conditions.

\subsubsection{Global force balance}

From literature it is known that six constraints should be satisfied to obtain global force balance [7]. Since four force balance conditions are found in the previous section, only two additional constraints are required for global force. These are

$$
d_{4}=\frac{m_{2} l_{4}}{m_{4} l_{2}} d_{2} \quad \gamma_{4}=\pi+\gamma_{2}
$$

Global moment balance of the five-bar mechanism is not possible without negative moments of inertia or counter rotations. This can be deducted from special kinematic conditions required for the dynamic balance of the four-bar mechanism [12].

\subsubsection{Reactionless paths}

Now that global force balance is established for the five-bar mechanism, one of the DOFs can be used to render moment balance, i.e. counteract the unbalance of the other DOF. In each pose we therefore have at least one dynamically balanced motion freedom. This motion freedom is computed using the null space operation $($ Eq. (51) and plotted for a grid of end-effector positions (Fig. 6). Numerical integration of this null-space operation yields reactionless paths. In the points where the instantaneous dynamic balance conditions are satisfied (here in $[0,0]$ ), multiple of these reactionless paths meet, locally allowing a two DOF dynamically balanced motion.

\subsection{Design of demonstrator}

Based on the previous dynamic balance properties a force balanced five-bar mechanism is designed with two intersecting reactionless paths to demonstrate the existence of instantaneous dynamic balance on a 6-axis force/torque sensor. The present dynamic balance conditions (Eq. (64) and Eq. (65) place conditions on $\mathbf{c}_{1}, \mathbf{c}_{3}, \mathbf{c}_{4}, g_{1}$, and $g_{3}$. This leaves a design freedom on kinematic parameters $\left(l_{0}-l_{4}\right)$ and the remaining inertial parameters $\left(m_{1}-m_{4}, \mathbf{c}_{2}, g_{2}\right.$ and $\left.g_{4}\right)$. These parameters can be chosen freely as long as all the masses and moments of inertia are positive. Here we aim to show that the current method opens a larger design freedom while with comparable reactionless paths as two mirror symmetrically connected dyads [33] or the DUAL V [19].

Therefore, the final design of the robot is chosen based on the following aspects: 1) The reactionless paths should be perpendicular and as straight as possible. 2) The mechanism must be constructible e.g. links should have sufficient stiffness. 3) Workspace should be sufficiently large. 4) Motor torques should be minimal. 5) The demonstrator should 
Table 2: The kinematic and inertial parameters of the Fuga I.

\begin{tabular}{lccc}
\hline Name & Symbol & Value & Unit \\
\hline Base width & $l_{0}$ & 400 & $\mathrm{~mm}$ \\
Upper arm length & $l_{1}, l_{3}$ & 190 & $\mathrm{~mm}$ \\
Lower arm length & $l_{2}, l_{4}$ & 180 & $\mathrm{~mm}$ \\
COM upper arm & $d_{1}, d_{3}$ & 69 & $\mathrm{~mm}$ \\
COM lower arm & $d_{2}, d_{3}$ & 140 & $\mathrm{~mm}$ \\
COM angle upper arm & $\gamma_{1}, \gamma_{3}$ & 150 & $\mathrm{deg}$ \\
COM angle lower arm & $\gamma_{2}, \gamma_{4}$ & 90 & $\mathrm{deg}$ \\
Mass upper arm & $m_{1}, m_{3}$ & 0.66 & $\mathrm{~kg}$ \\
Mass lower arm & $m_{2}, m_{4}$ & 0.21 & $\mathrm{~kg}$ \\
MOI upper arm & $g_{1}, g_{3}$ & 1.46 & $\mathrm{~g} \mathrm{~m}^{2}$ \\
MOI lower arm & $g_{2}, g_{4}$ & 1.66 & $\mathrm{~g} \mathrm{~m}^{2}$
\end{tabular}

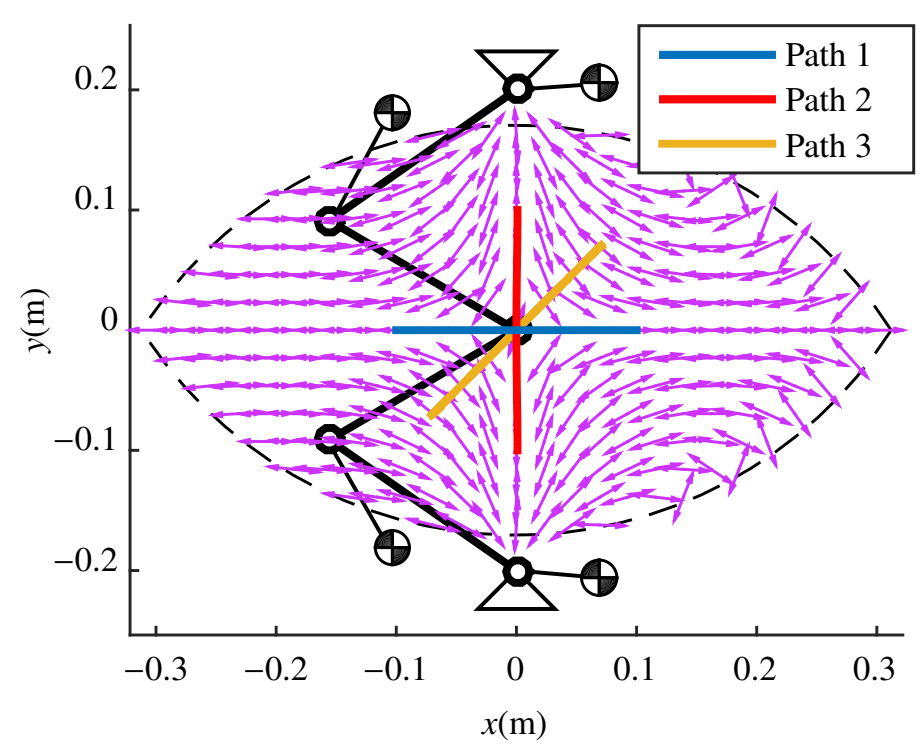

Figure 6: The resulting dynamically balanced mechanism the traces of reactionless paths. Path 1 and 2 are reactionless paths. Path 3 is the non moment balanced path. The dashed line denotes the workspace boundary. (For interpretation of the references to colour in this figure legend, the reader is referred to the web version of this article.)

fit on a 6-DOF force/torque sensor. 6) The center of mass of all links should be in a single plane to avoid out of plane torques.

Finally, an symmetric M shape design of the five-bar mechanism has been selected. The base joints are $400 \mathrm{~mm}$ apart. The upper arms and the lower arms have a length of $190 \mathrm{~mm}$ and $180 \mathrm{~mm}$, respectively. Refer to Table 2 for the other dimensions and design parameters. This gives the mechanism a singularity free workspace of with a minimal diameter of approximately $380 \mathrm{~mm}$. The instantaneously dynamically balanced pose lies in the middle of the workspace. The first reactionless path is along the symmetry line and the second is approximately along the base links (Fig. 6). This second reactionless path is an approximate straight line with a deviation $+/-0.5 \%$ (0.9 mm deviation over a stroke of $200 \mathrm{~mm}$ ). The exotically shaped upper arms are used for sufficient stiffness while providing the required moments of inertia. Direct drive brushless motors of Maxon EC-flat 90 are used. These have a peak torque of $7.4 \mathrm{Nm}$ and a moment of inertia of $3060 \mathrm{~g} \mathrm{~cm}^{2}$. A joint-space PID+ controller with computed torque control is used to steer the robot Fig. 7 shows the final design of the mechanism. 


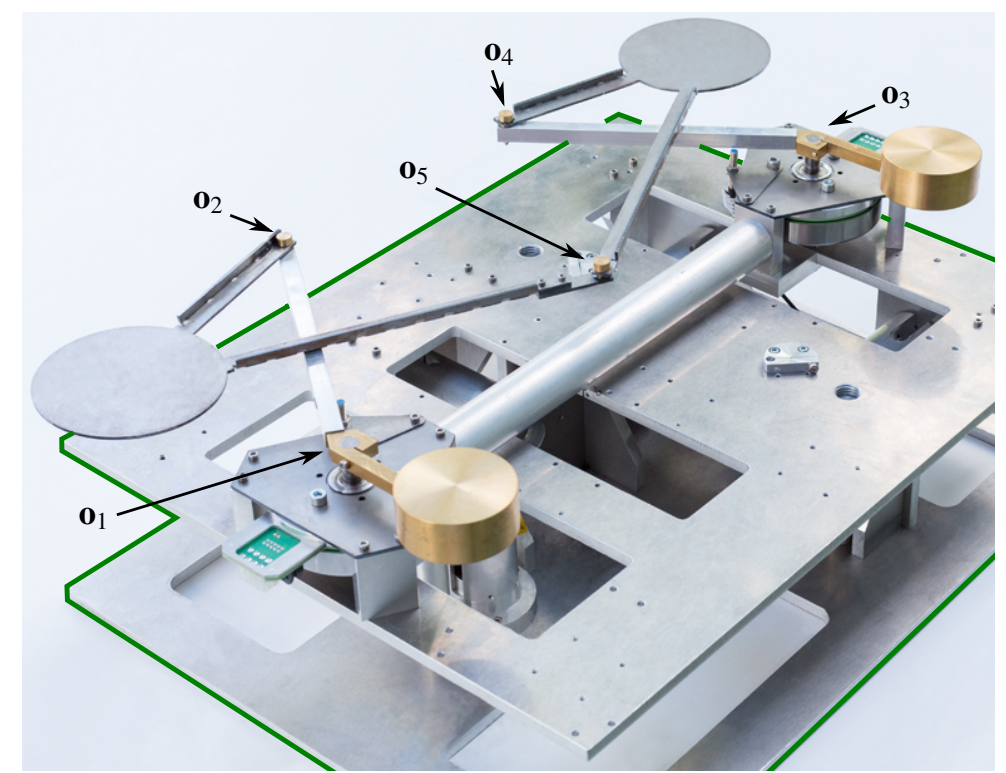

Figure 7: The 2-DOF dynamically balanced robot Fuga I mounted on a 6-DOF force/torque sensor (outlined) to measure the shaking forces and moments during motion.

\section{Evaluation of a dynamically balanced five-bar mechanism}

\subsection{Evaluation approach}

To evaluate the instantaneous dynamic balance of this demonstrator, the shaking forces and moments were measured over three paths, which all intersect in the instantaneously dynamically balanced pose. The first two paths are the reactionless paths and the third is a non-moment balanced path. As shown in Fig. 6 the first path is over the $x$-axis, the symmetry line of the mechanism. The second path is over the $y$-axis. The third and unbalanced motion is a diagonal over the workspace. To show that in the this pose the shaking moments are not affected by accelerations, a second order motion profile is chosen such that the joint accelerations switch in this pose. These paths have an equal length of $200 \mathrm{~mm}$ and the traveling time is $0.4 \mathrm{~s}$. This gives a maximum end-effector velocity and acceleration of 1 $\mathrm{m} / \mathrm{s}$ and $5 \mathrm{~m} / \mathrm{s}^{2}$, respectively. The shaking forces in the plane ( $x$ - and $y$-direction) and the shaking moment out of plane (z-direction) will be reported for these motions.

As the measurement setup and the mechanism showed an eigenfrequency around $27 \mathrm{~Hz}$, a lowpass filter with a cut-off of $10 \mathrm{~Hz}$ was applied to the force/torque measurements.

The mechanism is modeled in a multibody dynamics software. The measured joint angles are fed into this model to simulate and explain the resulting force and moment measurements. Firstly, this gives an estimation of the bearing forces at the base. In the perfectly force balanced case these forces completely cancel out. Therefore, the measured residual shaking forces are compared to the modeled internal forces for reference. Secondly, the modeled shaking moments are a measure for the shaking moments caused by path deviations. The difference between the modeled and the measured shaking moments gives a measure for construction and measurement errors.

\subsection{Results}

Fig. 9 shows the shaking moments and forces as measured for the three paths. The peak-to-peak shaking moment for the two reactionless paths are 0.06 and $0.04 \mathrm{Nm}$ respectively, for the unbalanced path this is $0.80 \mathrm{Nm}$. This corresponds to a reduction of $93 \%$ and $95 \%$ of the shaking moment with respect to the unbalanced path. The difference between the measured and modeled shaking moment is maximally $0.04,0.02$ and $0.10 \mathrm{Nm}$ for the three paths. In addition, the third trajectory shows instantaneous dynamic balance in the center of the workspace. In this pose, the switch of accelerations does not affect the shaking moments, as indicated by the arrows. 

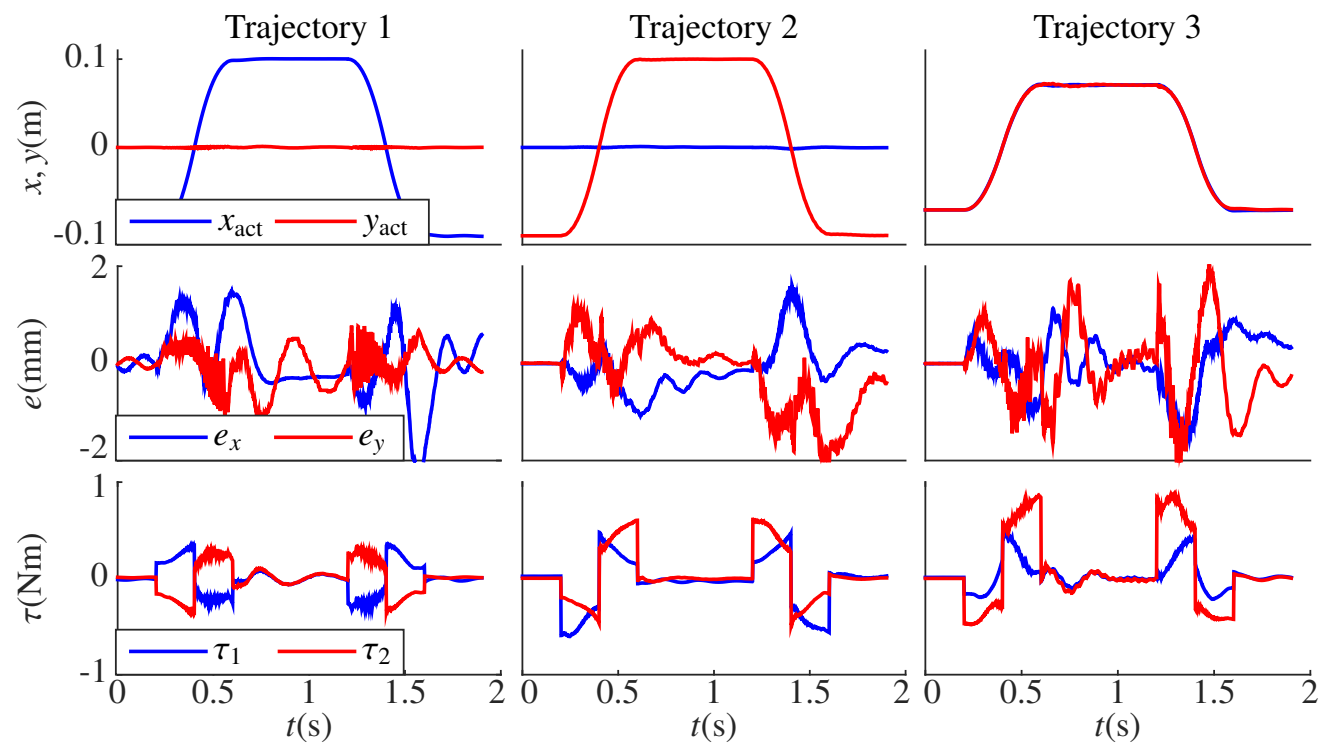

Figure 8: $x$ - and $y$-position of the end effector (top row), controller error in end-effector coordinates (middle row) and used motor torque (bottom row) for the three trajectories followed to evaluate force and moment balance. Trajectory 1 and 2 follow the two reactionless paths through the center of the workspace. Trajectory 3 follows the a non-moment balanced diagonal.
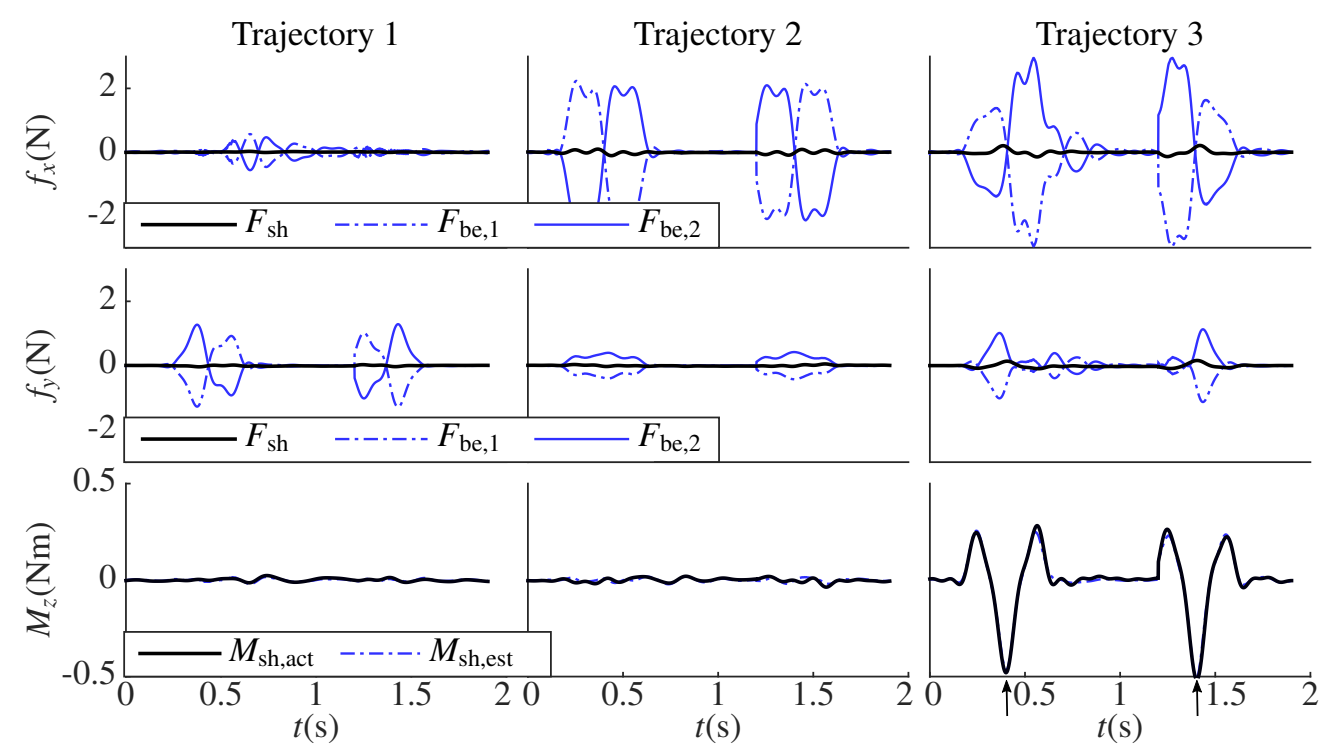

Figure 9: The shaking forces (top and middle) and moments (bottom) measured during the three trajectories are denoted with a thick solid line. For comparison the estimated bearing forces (top and middle) are given in thin solid and dashed lines. In the bottom row the estimated shaking moment based on the actual motion is shown with a dashed line. The arrows indicate where the acceleration switch. 
The shaking forces for the three paths have a peak-to-peak value of $0.26,0.11$ and $0.04 \mathrm{~N}$ respectively. When comparing these shaking forces to the estimated bearing forces, which are 5.93, 4.51, and $2.58 \mathrm{~N}$, it shows that approximately 96,98 , and $99 \%$ of the forces in the system cancel out.

Fig. 8 shows that the paths could be followed up to an end-effector accuracy of $2 \mathrm{~mm}$. This corresponds to $1 \%$ of the length of the path. This is computed by transforming the measured joint space error to the end-effector error under the assumption of perfect rigidity.

From the difference between the measured and the modeled shaking moments it is deduced that for the two reactionless paths $23 \%$ and $57 \%$ of the residual unbalance can be explained by controller inaccuracies causing the robot to deviate from the reactionless path. Other causes for the difference between measured and modeled shaking moments can be internal vibrations, production tolerances and inaccuracies. High frequency internal vibrations required low pass filtering of the force/torque sensor signal to eliminate the measured high frequency vibration. It is expected that this filtering influences the comparison between measured and modeled shaking forces and moments.

\section{Discussion}

Instantaneous dynamic balance can be found when the momentum wrenches in a mechanism sum to zero for all instantaneous velocities. By following a specific sequence through the chains, unique and solvable constraints are placed on the proximal part of the mechanism. From these conditions, the mass, COM and inertia matrix can be deduced for all cases. The remaining variables are the COM location along $\omega$ axis and principal moments of inertia $\mathbf{g}$. This fixes the mass, COM, and the orientation of the body. The positivity of mass and inertia, the triangle inequality put bounds on the choice of inertia matrices. This required that $\lambda_{t}$ should have same sign as $\omega^{\top} \mathbf{p}$ and that $\omega^{\top} \boldsymbol{\xi}_{c}>0$. In several special cases, additional requirements on $\mathbf{t}$ and $\mathbf{h}$ are to be satisfied (Table 1). These requirements are inherited over to bodies higher in the chain, limiting the choice of their inertial parameters. By satisfying these bounds, the mechanism can be constructed in theory. In practice, however, additional constraints on stiffness, shape, etc. will further limit the choice of inertial parameters.

The instantaneous dynamic balance conditions can be found for any robot and do not require the solution or inspection of the loop closure equations. It merely relies on the differential kinematics, which are readily available through screw theory.

To extend the instantaneous dynamic balance to global dynamic balance, additional conditions are to be met. It requires not only that the momentum is zero in a certain pose, but also in all other poses. Harmonizing this step is beyond the scope of this paper.

The Fuga I was constructed to demonstrate that the presented method results in instantaneous dynamic balance. Although its unusual mass distribution is not likely to be used in industry, its dynamic balanced paths are comparable to that of the DUAL V [19], yet is achieved through a design with two instead of four motors. Also, the COM does not have to lie on the line through the pivots, and the upper and lower link lengths can differ. This shows that with this method, novel dynamic balance solutions can found for mechanisms with reactionless paths, and that the location of their intersection points can be chosen freely. The shape of the reactionless paths is determined by the remaining parameters, the choice of which is outside the scope of this paper. Here an iterative approach was adopted, which resulted in perpendicular, approximate straight paths.

The dynamic balance measures as reported here are dependent on the cycle time, motion profiles and the choice of paths. Due to the velocity dependent terms in the shaking forces and moments and hence in the dynamic balance measures. Here, the worst and best trajectories possible for this setup are compared. These are therefore regarded as representative of the robot.

\section{Conclusion}

For the first time screw theory has been applied to derive a subset of the dynamic balance conditions for any planar or spatial, single or multi-DOF mechanism in a general, geometrical manner, without requiring tedious manipulation of equations.

The presented screw-based dynamic balance method provides and solves six conditions per DOF in spatial case, and three per DOF in the planar case. These conditions yield instantaneous dynamic balance, which is a prerequisite 
for global dynamic balance. This is interpreted as pose, in which multiple reactionless paths intersect. The freedom and bounds on the choice of masses, centers of mass, and moments of inertia where interpreted in a geometric manner.

This method is applied to a planar five-bar mechanism and gave six instantaneous dynamic balance conditions; four out of six conditions for global force balance and two conditions on instantaneous moment balance. The mechanism was designed such that two reactionless paths intersect in the middle of the workspace and are approximately perpendicular straight lines. Measurements and simulations showed that the shaking forces where at least $96 \%$ lower then the internal bearing forces, indicating force balance. The measurements also confirmed the existence of two intersecting reactionless paths. When comparing the non-moment balanced paths with the two reactionless paths a shaking moment reduction in the order of $95 \%$ was achieved. This shows the existence of a instantaneously dynamically balanced pose in the middle of the workspace.

\section{References}

[1] J. Karidis, G. McVicker, J. Pawletko, L. Zai, M. Goldowsky, R. Brown, R. Comulada, The Hummingbird minipositioner-providing three-axis motion at $50 \mathrm{~g}$ 's with low reactions, Proceedings 1992 IEEE International Conference on Robotics and Automation (1992) 685-692.

[2] G. G. Lowen, F. R. Tepper, R. S. Berkof, Balancing of linkages_an update, Mechanism and Machine Theory 18 (3) (1983) 213-220.

[3] V. H. Arakelian, M. R. Smith, Shaking Force and Shaking Moment Balancing of Mechanisms: A Historical Review With New Examples, Journal of Mechanical Design 127 (2) (2005) 334

[4] V. Van der Wijk, J. L. Herder, B. Demeulenaere, Comparison of Various Dynamic Balancing Principles Regarding Additional Mass and Additional Inertia, Journal of Mechanisms and Robotics 1 (4) (2009) 041006.

[5] J. Wiederrich, B. Roth, Momentum Balancing of Four-Bar Linkages, Journal of Engineering for Industry - Transactions of the ASME 98 (4) (1976) 1289-1295.

[6] R. S. Berkof, G. G. Lowen, A new method for completely force balancing simple linkages, Journal of Engineering for Industry 91 (1) (1969) 21.

[7] P. Ouyang, Q. Li, W. Zhang, Integrated design of robotic mechanisms for force balancing and trajectory tracking, Mechatronics 13 (8-9) (2003) 887-905.

[8] S. Foucault, C. M. Gosselin, On the development of a planar 3-DOF reactionless parallel mechanism, ASME 2002 International Design Engineering Technical Conferences and Computers and Information in Engineering Conference (2002) 1-9.

[9] S. Briot, V. Arakelian, Complete shaking force and shaking moment balancing of in-line four-bar linkages by adding a class-two RRR or RRP Assur group, Mechanism and Machine Theory 57 (2012) 13-26.

[10] T. Laliberté, C. M. Gosselin, Synthesis, optimization and experimental validation of reactionless two-DOF parallel mechanisms using countermechanisms, Meccanica 51 (12) (2016) 3211-3225.

[11] C. Bagci, Complete Shaking Force and Shaking Moment Balancing of Link Mechanisms Using Balancing Idler Loops, Journal of Mechanical Design 104 (2) (1982) 482-493.

[12] R. Ricard, C. M. Gosselin, ON THE DEVELOPMENT OF REACTIONLESS PARALLEL MANIPULATORS, in: ASME 2000 Design Engineering Technical Conferences and Computers and Information in Engineering Conference, Vol. 1, Baltimore, Maryland, 2000, pp. $1-10$.

[13] C. M. Gosselin, B. Moore, J. Schicho, Dynamic balancing of planar mechanisms using toric geometry, Journal of Symbolic Computation 44 (9) (2009) 1346-1358.

[14] B. Moore, J. Schicho, C. M. Gosselin, Dynamic balancing of spherical 4R linkages, Journal of Mechanisms and Robotics 2 (2) (2010) 021002.

[15] Y. Wu, C. M. Gosselin, Synthesis of Reactionless Spatial 3-DoF and 6-DoF Mechanisms without Separate Counter-Rotations, The International Journal of Robotics Research 23 (6) (2004) 625-642.

[16] V. Van der Wijk, J. L. Herder, Guidelines for low mass and low inertia dynamic balancing of mechanisms and robotics, Advances in Robotics Research (2009) 21-30.

[17] O. Fischer, Theoretische Grundlagen für eine Mechanik der lebenden Körper, Teubner, Leipzig, 1906.

[18] V. Van der Wijk, J. L. Herder, Synthesis method for linkages with center of mass at invariant link point — Pantograph based mechanisms, Mechanism and Machine Theory 48 (2011) 15-28.

[19] V. Van der Wijk, S. Krut, F. Pierrot, J. L. Herder, Design and experimental evaluation of a dynamically balanced redundant planar 4-RRR parallel manipulator, The International Journal of Robotics Research 32 (6) (2013) 744-759.

[20] V. Van der Wijk, Shaking-moment balancing of mechanisms with principal vectors and momentum, Frontiers of Mechanical Engineering 8 (1) (2013) 10-16.

[21] E. Papadopoulos, A. Abu-Abed, Design and motion planning for a zero-reaction manipulator, Proceedings of the 1994 IEEE International Conference on Robotics and Automation 7 (1994) 1554-1559.

[22] K. Yoshida, K. Hashizume, S. Abiko, Zero reaction maneuver: flight validation with ETS-VII space robot and extension to kinematically redundant arm, in: International Conference on Robotics and Automation, Seoul, Korea, 2001, pp. 441-446.

[23] I. S. Kochev, Full shaking moment balancing of planar linkages by a prescribed input speed fluctuation, Mechanism and Machine Theory 25 (4) (1990) 459-466.

[24] J. J. De Jong, J. Van Dijk, J. L. Herder, A Screw-Based Dynamic Balancing Approach, Applied to a 5-Bar Mechanism, in: J. Lenarcic, J.-P. Merlet (Eds.), Advances in Robot Kinematics 2016. Springer Proceedings in Advanced Robotics, Springer, Cham, 2018 , pp. 33-41.

[25] R. S. Ball, A treatise on the theory of screws, Cambridge University Press, UK, Cambridge, 1900.

[26] M. Zoppi, D. Zlatanov, R. Molfino, On the velocity analysis of interconnected chains mechanisms, Mechanism and Machine Theory 41 (11) (2006) 1346-1358. 
[27] X. Kong, C. M. Gosselin, Type synthesis of parallel mechanisms, Springer, Berlin-Heidelberg, 2007.

[28] J. Selig, Applying screw theory to robot dynamics, Journal of Applied Mathematics and Mechanics 55 (3) (1991) 159-167.

[29] G. R. Pennock, P. J. Meehan, Geometric Insight Into the Dynamics of a Rigid Body Using the Spatial Triangle of Screws, Journal of Mechanical Design 124 (4) (2002) 684

[30] R. Featherstone, Rigid Body Dynamics Algorithms, Springer US, Boston, MA, 2008.

[31] J. M. Selig, D. Martins, On the line geometry of rigid-body inertia, Acta Mechanica 225 (11) (2014) 3073-3101.

[32] R. M. Murray, Z. Li, S. S. Sastry, A Mathematical Introduction to Robotic Manipulation, Vol. 29, 1994. arXiv:0712.0689

[33] S. Briot, I. a. Bonev, C. M. Gosselin, V. Arakelian, Complete shaking force and shaking moment balancing of planar parallel manipulators with prismatic pairs, Proceedings of the Institution of Mechanical Engineers, Part K: Journal of Multi-body Dynamics 223 (1) (2009) 43-52. 